\title{
Études/Inuit/Studies
}

\section{Le peuple yupik et ses voisins en Tchoukotka: huit décennies de changements accélérés}

\section{The Yupik people and its neighbours in Chukotka: Eight decades of rapid changes}

\section{Yvon Csonka}

Volume 31, numéro 1-2, 2007

Tchoukotka

Chukotka

URI : https://id.erudit.org/iderudit/019713ar

DOI : https://doi.org/10.7202/019713ar

Aller au sommaire du numéro

Éditeur(s)

Association Inuksiutiit Katimajiit Inc.

Centre interuniversitaire d'études et de recherches autochtones (CIÉRA)

ISSN

0701-1008 (imprimé)

1708-5268 (numérique)

Découvrir la revue

Citer ce document

Csonka, Y. (2007). Le peuple yupik et ses voisins en Tchoukotka: huit décennies de changements accélérés. Études/Inuit/Studies, 31(1-2), 7-37.

https://doi.org/10.7202/019713ar 


\section{Le peuple yupik et ses voisins en Tchoukotka: huit décennies de changements accélérés}

Rédacteur invité:

Yvon Csonka*

\section{Introduction}

Études/Inuit/Studies a régulièrement publié des articles sur le peuple yupik de la Tchoukotka depuis son cinquième volume, en 1981, qui présentait des contributions de Boris Chichlo et d'Igor Krupnik, jusqu'à tout récemment, en 2005, avec le volume double, «Préserver la langue et les savoirs» ${ }^{1}$. Mais ce numéro thématique de la revue est cependant le premier à présenter une vue aussi étendue des peuples de la Tchoukotka. La première impulsion à l'origine de ce volume naquit nulle part ailleurs qu'au Groenland, au cours d'une réunion du bureau de rédaction de la revue en 2002, lorsqu'un de ses membres, qui fait également partie du conseil d'administration de l'Inuit Circumpolar Council (ICC), Carl Christian Olsen (Puju), émit l'idée d'un numéro spécial sur la Tchoukotka. Compte tenu de mes précédentes recherches et des liens familiaux que j'entretiens dans cette région, en plus de mon réseau de collègues, j'acceptai de grand cœur le rôle de rédacteur invité.

\section{Yupik, Tchouktches et «Tchoukotka»}

En Russie, le terme Eskimosy (Esquimaux) demeure l'(auto)-désignation acceptée et officielle des peuples apparentés aux populations inuit et yup'ik du Nouveau Monde. Parmi d'autres désignations, on trouve les termes «Esquimau asiatique» — utilisé par Bogoras et par les chercheurs russes qui lui ont succédé dans les dernières décennies du 20e siècle - et les termes «Esquimau sibérien» ou "Yupik sibérien», des plus usuels aux États-Unis ${ }^{2}$. Yupik (plur. Yupiget, 'véritable être humain') est l'auto-désignation vernaculaire des groupes parlant le yupik de la Sibérie Centrale (YSC), de la Tchoukotka et de l'île St-Laurent, en Alaska, qui fait partie des États-Unis (Figures 1 et 2). Les locuteurs du YSC et leurs descendants constituent la majorité des Eskimosy

Département d'histoire culturelle et sociale, Ilisimatusarfik-Université du Groenland, Box 1061, DK3900 Nuuk, Groenland.csonka@greennet.gl

I Deux numéros de la revue furent partiellement consacrés à la Tchoukotka et à ses peuples: vol. 5(2) (1981) intitulé «Yuit-Esquimaux asiatiques», et vol. 17(2) (1993) «Tchoukotka, Nunavut, Nunavik».

2 Dans un article de référence, Hughes (1984: 259) considère les Esquimaux de Sibérie comme un sousgroupe des Esquimaux asiatiques excluant les insulaires de St-Laurent. Le nom de Yuit (sing. Yuk, 'personne') que l'on trouve parfois dans les écrits sur ce sujet, n'était pas utilisé comme autodésignation. 
vivant en Russie. On ne doit pas les confondre avec les Yupiit (sing. Yup'ik) de l'ouest de l'Alaska qui leur sont apparentés sur le plan linguistique ${ }^{3}$. La Tchoukotka est, ou était, le foyer d'autres populations d'ascendance esquimaude. Les Nevuqaghmiit, qui furent expulsés de Naukan en 1958, (Figure 3; Chichlo 1981; Krupnik et Chlenov, dans ce volume) parlent un dialecte apparenté à celui des Yup'ik du centre de l'Alaska et, dans leur langue, la désignation des Esquimaux non-Inuit, y compris eux-mêmes, est Yupiit (sing. Yupik). Mikhail Chlenov, l'un des contributeurs de ce volume, surnomma un jour Naukan «la troisième des îles Diomède», en décrivant sa situation géographique et culturelle, isolée du reste de la Péninsule des Tchouktches par la chaîne des monts Dezhnev (Chlenov 1988: 71); et ces derniers siècles, les îles Diomède ont constitué une voie d'accès vers l'Asie pour les peuples de l'Alaska et leur influence, d'abord les Yupiit, puis, plus tard, les Inupiat. En Russie, le terme Eskimosy désigne également un petit groupe de descendants des Inupiat déplacés de l'île Grande Diomède en 1948, tout autant que d'anciens locuteurs de la langue aujourd'hui éteinte des Esquimaux Sirenikski, qui n'était ni yupik ni inupiaq. Ainsi, les termes Yupiget, Yupiit et «Esquimaux» ne désignent pas tout à fait les mêmes populations. Nous avons laissé aux contributeurs de ce volume une certaine flexibilité dans leur usage des désignations, à des fins de précision, et pour ne pas contrevenir aux usages locaux.

Sous certains aspects, la Tchoukotka est une région marginale du monde inuit d'aujourd'hui. Les Eskimosy, comptant environ 1600 personnes, ne constituent qu'une petite minorité dans leur propre région, d'autant plus réduite si on la replace à l'échelle de la gigantesque Russie ${ }^{4}$. Lorsque l'Alaska fut vendu aux États-Unis, en 1867, les Yupiget furent scindés entre deux pays aux systèmes politiques, culturels et idéologiques très différents. La séparation fut définitive durant la Guerre froide (19481988) qui éleva des barrières entre les peuples de la région, empêchant les interactions, la liberté des déplacements, les liens familiaux et maritaux qui avaient autrefois prospéré pendant des générations. La Tchoukotka, en tant que région, fut coupée du reste du monde pendant une grande partie du 20e siècle, en raison des développements politiques en Russie. La région elle-même (Figure 2) est depuis longtemps désignée d'après le nom des voisins plus nombreux des Yupik, les Tchouktches ${ }^{5}$. L'entité administrative actuelle fut instaurée en 1930 sous le nom de «Région Nationale Tchouktche» (okrug en russe), et elle fut rebaptisée «Région Autonome Tchouktche»

Les termes Yupik en Asie, et Yup'ik en Alaska, au singulier, sont d'usage courant dans les publications en tant que forme adjectivale invariable, et souvent également en tant que pluriel du nom, là où la tradition a imposé le pluriel Inuit comme adjectif invariable, sauf pour désigner un individu (Dorais 2004: 156). La politique éditoriale de la présente revue n'accepte pas le terme Yupik dans le sens pluriel.

4 Selon le recensement de 2002 en Russie, il y avait 1534 «Esquimaux» en Tchoukotka même, et 216 de plus vivant dans d'autres parties de la Fédération de Russie (Goskomstat Rossii 2004). Les Eskimosy représentent environ le dixième de la population autochtone de la Tchoukotka, et ils ne constituent la majorité de la population que dans un seul village, Novoye Chaplino.

5 La désignation Chukchi (qui est un pluriel en russe), est censée dériver de l'auto-désignation des éleveurs de rennes à l'intérieur de ce peuple, tandis que les Tchouktches, tant du bord de mer que de l'intérieur se désignent eux-mêmes sous le nom de Lyg'oravetl'an ('le peuple par excellence'). En 2002, il y avait 12622 Tchouktches en Tchoukotka même (Goskomstat Rossii 2004). 


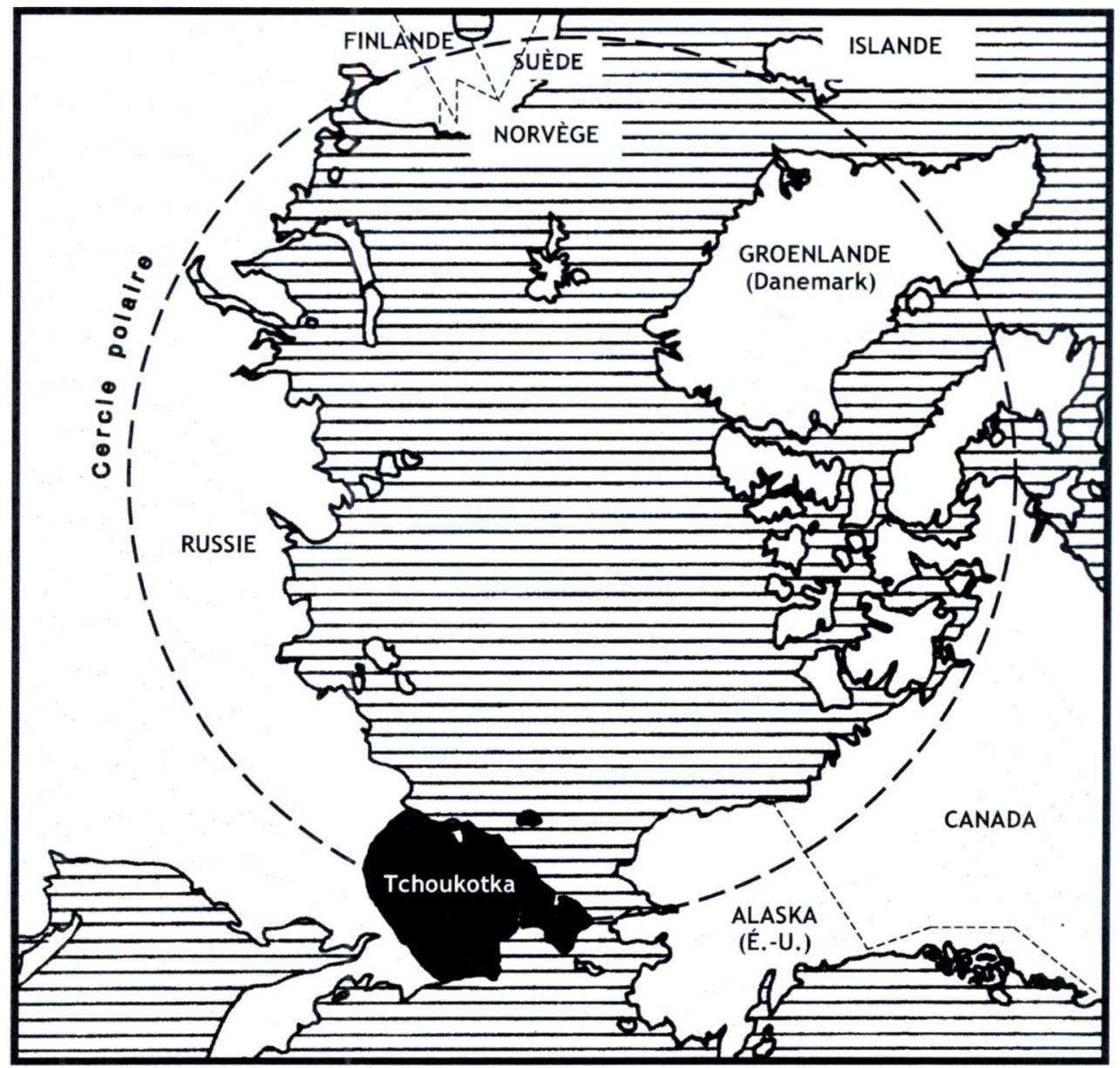

Figure 1. Carte de l'Arctique montrant la localisation de la Tchoukotka. Carte de Bent Nielsen. 


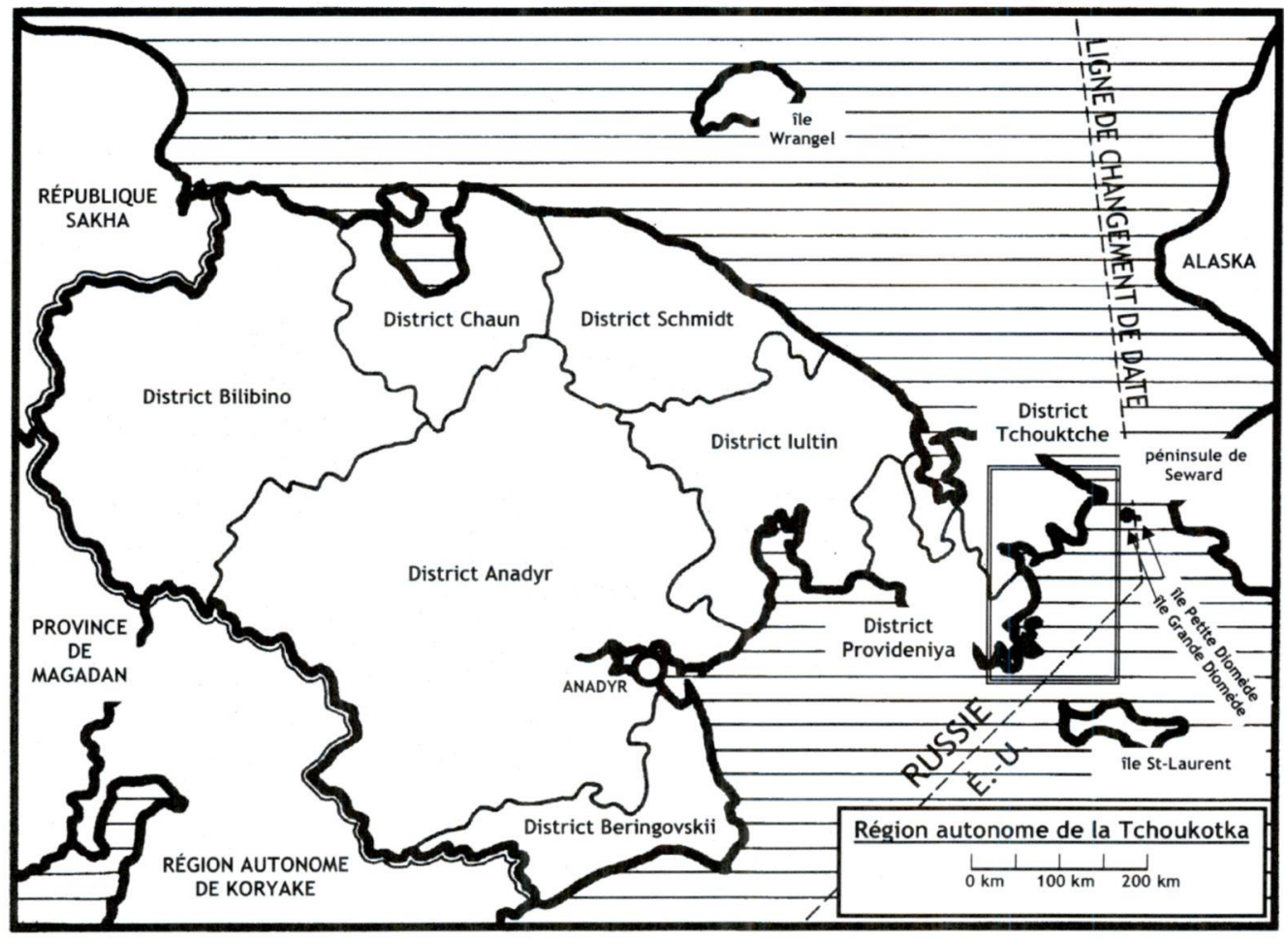

Figure 2. Carte des districts et des territoires adjacents de la Tchoukotka. L'encart montre la localisation de la Figure 3. Carte de Bent Nielsen. 


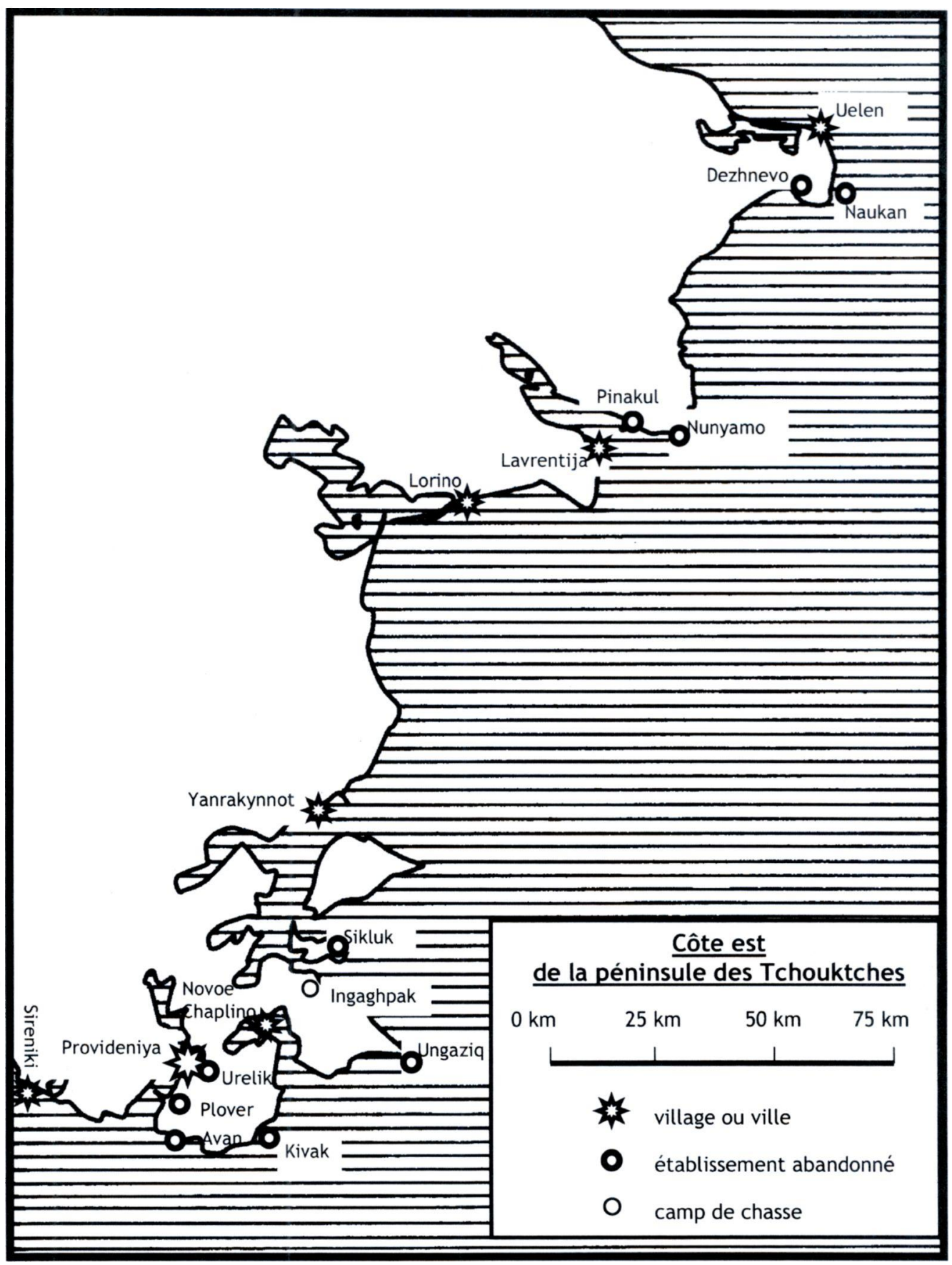

Figure 3. Carte de la côte est de la péninsule des Tchouktches. Carte de Bent Nielsen. 
en 1980 (Chukotskii avtonomnyi okrug), abandonnant ainsi la référence ethnique ${ }^{6}$. Cela reste son nom officiel, ordinairement abrégé en russe sous la forme «Tchoukotka»et, par extension, en français aussi. Ce nom de Tchoukotka est devenu d'usage si courant qu'il s'est imposé dans l'expression française «Région autonome de Tchoukotka». Cette région, cependant, reste au cœur de l'histoire ancienne des Inuit, puisqu'elle fut le berceau des développements néoesquimaux les plus anciens il y a environ 2000 ans, et qu'elle fut en grande partie à l'origine de la culture de Thulé qui se diffusa dans l'Arctique canadien et au Groenland un millénaire plus tard (voir par exemple Csonka 2003; Dumond 1987; Gulløv et McGhee 2006; Plumet 1996). Cette dernière constitue la toile de fond de toute l'histoire subséquente des cultures inuit et yupik.

En Asie, moins encore que dans toute autre région où ils s'installèrent, les peuples esquimaux/yupik n'étaient pas isolés des populations environnantes. Ils étaient en contact constant avec leurs différents voisins, qui leur transmirent de nombreux éléments culturels venus d'ailleurs en Sibérie et en Asie, ainsi qu'avec les Inupiat et les Yupiit de l'Alaska. La population esquimaude d'Asie était historiquement très diversifiée, parlant plusieurs langues et dialectes distincts. Au fil des temps, les échanges entre les populations furent intenses, à travers la traite, le mariage, le comariage, l'intégration de prisonniers de guerre sous forme d'épouses ou d'esclaves, et divers degrés de multilinguisme. Les chercheurs russes pensent que la population tchouktche maritime est issue d'Esquimaux «tchouktchifiés», qui adoptèrent la langue et certaines des coutumes de leurs voisins de l'intérieur des terres, sans abandonner leurs habitudes économiques et leurs schèmes d'établissement d'origine (Arutiunov et al. 1982; Arutiunov et Sergeev 1975; Bogoras 1904-1909). Les Esquimaux préhistoriques et historiques se sont également, à certaines époques, répandus plus loin qu'aujourd'hui le long des rivages arctique et pacifique de la Tchoukotka. Des rencontres culturelles tout aussi complexes se sont poursuivies tout au long du 20e siècle, avec l'immigration massive de nouveaux venus de nombreuses nationalités soviétiques, et les politiques soviétiques consistant à rassembler différentes populations dans de grands établissements multiethniques où elles étaient incitées aux mariages mixtes, au multilinguisme et à une acculturation rapide. De fait, à l'époque soviétique, les peuples autochtones devinrent minoritaires en Tchoukotka: ils représentaient $96 \%$ de la population de la région en 1926, mais moins de $10 \%$ en 1989. En raison du départ massif des immigrants, leur part dans la population totale est depuis remontée à près d'un tiers en $2002^{7}$.

6 À ne pas confondre avec l'appendice géographique appelé la Péninsule des Tchouktches (Chukotskii Poluostrov), qui se restreint approximativement aux deux districts de la Tchoukotka les plus près du Détroit de Béring (Figure. 2). La plus grande partie de la population yupik de la Tchoukotka vit sur la côte est de la Péninsule des Tchouktches (Figure 3).

7 L'histoire et l'ethnographie de la population immigrée en Tchoukotka à l'époque soviétique et postsoviétique ont été récemment décrites en détail dans l'excellent ouvrage de Thompson (à paraître), qui est un incontournable pour quiconque souhaite comprendre les développements politiques et sociaux en Tchoukotka. Les questions d'ethnicité dans les habitats mixtes à la fin de l'époque soviétique ont été décrites par Kerttula (1997, entre autres) et par Schindler (1992, 1997), et pour l'époque post-soviétique par Nielsen (2005) et par Vakhtin et Krupnik (1999). 


\section{Recherche en Tchoukotka après la Perestroïka}

Ce numéro thématique coïncide avec le vingtième anniversaire de ce que l'on a appelé le «Vol de l'amitié», parti de Nome en Alaska pour se poser à Provideniya, en Tchoukotka. L'avion transportant des hommes politiques de l'Alaska, des fonctionnaires, des journalistes et des aînés yupik de l'île St-Laurent qui atterrit en juin 1988 à Provideniya, au coeur du territoire yupik soviétique, annonça la fin des quatre décennies de Guerre froide dans la région du Détroit de Béring qui, littéralement, rétablit les contacts entre la Tchoukotka et le reste du monde (Krauss 1994). Le mois suivant, une délégation de l'ICC vint également visiter la région. Au cours de la même période, l'exposition "Crossroads of Continents» ('Au carrefour des continents') (1988), ainsi que de nombreuses publications universitaires subséquentes, remirent en lumière le concept de $\mathrm{l}^{\text {' }}$ "aire culturelle du Pacifique Nord» ${ }^{8}$ et incitèrent par là même à un renouveau des collaborations dans la recherche intercontinentale (Fitzhugh et Chaussonnet 1994; Fitzhugh et Crowell 1988; Krupnik et Fitzhugh 2001).

Depuis la fin de la Guerre froide, de nombreux chercheurs de la région, du reste de la Russie et d'autres pays, ont porté le champ de la recherche en sciences humaines et sociales en Tchoukotka à un niveau que l'on n'avait plus vu depuis des décennies. Tous furent confrontés à de considérables difficultés logistiques et administratives. Rien ne témoigne plus tragiquement de ces difficultés que les deux accidents mortels qui nous enlevèrent certains de nos collègues. En mai 1993, à la fin de l'expédition «Transsibéring», un hélicoptère s'écrasa sur la banquise près d'Enurmino, tuant huit des 21 passagers parmi lesquels se trouvaient l'ethnologue Galina Gracheva, des journalistes russes et étrangers et un représentant des organismes subventionnaires de l'expédition (Kunstkamera n.d.) ${ }^{9}$. En septembre 1995, plusieurs collègues russes et étrangers travaillant en Tchoukotka - Aleksandr Pika, Steven McNabb, Richard Condon, William Richards - périrent, en même temps que des personnes originaires du lieu, lorsqu'un bateau non ponté chavira au large de Sireniki, dans des conditions jamais élucidées (Krupnik et Fitzhugh 1995; Stern 1997: 1). Ainsi, après une courte période initiale d'échanges enthousiastes, la Tchoukotka redevint la région lointaine, difficile d'accès et quasi-interdite qu'elle est encore aujourd'hui.

8 La comparaison des cultures de la bordure nord du Pacifique était le principe qui guidait l'expédition Jesup du Pacifique Nord (1897-1902) que dirigea Franz Boas, du Muséum d'histoire naturelle de New York, et qui produisit des contributions magistrales dans les domaines de l'ethnographie, du folklore et du langage, en particulier celles de Waldemar Bogoras sur les Tchouktches et les Yupiget (1904-1909, 1913).

9 Des fonds privés recueillis en France et en Suisse à la suite de cette tragédie permirent à l'organisation non gouvernementale Médecins du Monde de mener à bien des projets humanitaires visant à soulager le sort des Autochtones de la Tchoukotka pendant le reste des années 1990. Ces efforts humanitaires furent à peine tolérés par l'administration de la Région. 


\section{Changement et continuité chez les peuples de la Tchoukotka}

Considérant le contexte de longue durée des intenses contacts culturels en Tchoukotka, il semble justifié, dans ce numéro thématique, d'éviter de limiter les contributions aux seuls peuples yupik mais, au contraire, d'ouvrir la discussion à ce qui fut un élément central de leur histoire récente, l'incorporation collective des peuples autochtones de la Tchoukotka à l'État soviétique, puis post-soviétique. Sous de nombreux aspects, les bouleversements du 20e siècle ont dissocié les Yupiit et les Yupiget de la Tchoukotka de leurs voisins inuit et yup'ik de l'Alaska et d'ailleurs. Mais les lecteurs ayant une bonne connaissance des conditions et de l'histoire des peuples inuit d'autres pays arctiques reconnaîtront aisément de nombreux phénomènes, et de nombreuses tendances sociales leur seront familières; ils réaliseront que si certaines sont de degré différent, elles sont de type semblable.

Les histoires démographiques comparées des différentes régions inuit nous donnent une indication des conditions de vie médiocres des peuples autochtones de la Tchoukotka: de 1926 à 2002, la population yupik n'a augmenté que de $19 \%$, celle des Tchouktches de $16 \%$. Par contraste, au cours de périodes comparables, les Groenlandais sont devenus deux fois plus nombreux, tandis que la population des Inuit du Canada a été multipliée par six environ ${ }^{10}$. L'évolution démographique des populations yupik du passé a été décrite comme très dynamique, restreinte périodiquement seulement par des facteurs environnementaux (Krupnik 1993: chap. 7). Mais la quasi stagnation (avec de légères oscillations) de la population tout au long du $20 \mathrm{e}$ siècle doit s'expliquer par d'autres contraintes que celles de l'environnement. Les changements dans les affiliations ethniques (ou leurs définitions), l'assimilation ou les déficiences des statistiques ne sont que des facteurs d'explication secondaires. L'alcoolisme endémique, l'insuffisance des soins médicaux, les pauvres conditions sanitaires des mères et de leurs enfants, et les taux de morts violentes extrêmement élevés, liés aux problèmes sociaux générés par les changements imposés, sont les facteurs qu'invoquent les articles de référence des spécialistes russes remontant à l'époque de la Perestroïka (Bogoyavlinsky 1996[1989]; Krupnik 1987; Pika 1987; Pika et Prokhorov 1988; Vakhtin 1992) et se poursuivant jusqu'à nos jours (voir contributions d'Abryutina dans ce volume).

Les articles de ce volume nous offrent, sous forme condensée, un panorama de la diversité de la recherche en Tchoukotka au cours des 10 ou 20 dernières années, ainsi que les résultats de contributions antérieures au champ des études yupik, dans plusieurs disciplines ${ }^{11}$. Les auteurs proviennent de la Tchoukotka elle-même, du reste de la Russie, de différents pays européens et de l'Amérique du Nord. Beaucoup parmi ces contributions documentent et analysent non pas tant des aspects des cultures et des

10 Estimation de l'auteur se basant sur les données de recensement (dans Chichlo 1993; Choinière et Robitaille 1983; Grønlands statistik 2007). Ces estimations ne tiennent pas compte de l'émigration des populations inuit et yupik hors de leur région. Cette émigration est proportionnellement plus élevée au Groenland et au Canada qu'en Russie.

11 On ne trouvera pas de contribution à l'archéologie dans ce volume. Pour des publications récentes portant sur la préhistoire néoesquimaude de la Tchoukotka, voir par exemple Bronshtein et al. (2007); Csonka (2003); Dumond et Bland (2002) et Krupnik et al. (2006). 
sociétés traditionnelles autochtones en tant que tels, mais plutôt les impacts des régimes soviétique, puis plus tard, post-soviétique, tout autant que les réactions et les adaptations des Autochtones à ceux-ci. Cet angle de vue ne découle pas d'un choix éditorial délibéré; il constitue simplement la preuve de l'amplitude de ces impacts, qui ont affecté tous les peuples autochtones de la région, et il était inévitable que l'attention de nombreux chercheurs en ait été frappée et qu'ils en aient fait leur sujet d'étude. Les changements de l'époque post-soviétique, dont l'extrême rapidité avait déjà été relevée dans les années 1990 (e.g., Csonka 1998: 24; Vakhtin et Krupnik 1999; Krupnik et Vakhtin 2002: 7, 32), se poursuivent sans répit. Nous nous trouvons donc bien loin de l'illusion d'un présent ethnographique intemporel, et l'ordre des articles de ce volume reflète cette perspective liée au temps.

Dans la section «Documents», Abryutina nous présente un survol général de l'histoire des «peuples autochtones de la Tchoukotka» du point de vue d'une personne qui en fait partie. Ce texte devait à l'origine constituer le prologue de sa présentation sur la partie consacrée à la Tchoukotka dans le projet SLiCA (Survey of Living Conditions in the Arctic ['Enquête sur les conditions de vie dans l'Arctique']) qui fut entrepris sous sa direction en 2005, et qui apparaît ici parmi les notes de recherche. Les comparaisons des résultats produits par le projet SLiCA dans les différentes régions du monde inuit, qu'elle présente ici, démontrent à quel point les Autochtones de la Tchoukotka sont les moins bien lotis, et qu'ils en sont conscients, en termes de santé, de consommation de nourriture locale, de logement et de sécurité économique. Toujours dans l'esprit de cette vision de l'intérieur, elle a choisi de se concentrer sur le contexte local et les commentaires libres des répondants, tandis que l'on peut trouver d'autres tableaux comparatifs dans l'ouvrage récent qui présente les résultats de cette enquête internationale (Poppel et al. 2007).

Des données historiques orales collectées dans les années 1990 permettent à Schweitzer et Golovko de documenter un épisode intrigant d'une régénérescence religieuse autochtone à Naukan au début des années 1930. Dans leur contribution à ce volume, les auteurs décrivent le contexte des rencontres entre cultures et religions au cours de la période où se situe cet événement, et relient ce dernier, dans une analyse théorique pénétrante, aux tensions provoquées par la transition du colonialisme américain au colonialisme russe, et en particulier aux changements imposés sous l'autorité des Soviets. La résistance à Naukan fut de courte durée: le village fut fermé par les autorités soviétiques en 1958 et son accès interdit. Les pionniers de l'ethnographie yupik, Krupnik et Chlenov, menaient déjà des enquêtes de terrain et des entrevues d'histoire orale auprès des personnes relocalisées dans les années 1970 et 1980. Ici, ils décrivent la manière dont les schèmes d'établissement yupik en Tchoukotka furent détruits à la fin des années 1950, et cela, pour la première fois, dans les propres mots des personnes déplacées, et de leur point de vue (parmi les contributions antérieures, dans cette revue, au sujet des déplacements de population de Naukan, on consultera Chichlo [1981] et Robert-Lamblin [1993]). Bien que les relocalisations aient affecté moins de 1000 personnes et qu'elles n'aient pas impliqué de grandes distances, les auteurs montrent qu'elles ont eu un impact particulièrement destructeur sur les cultures et les sociétés yupik. 
L'histoire de l'atelier de gravure et de sculpture du village d'Uelen, situé tout près, telle que la raconte Bronshtein, commence à peu près en même temps que le mouvement de revitalisation de Naukan, en 1931, et s'est épanouie jusqu'à aujourd'hui. L'auteur insiste sur la continuité de la culture spirituelle et de l'expression artistique autochtones, en dépit du fait évident que cette entreprise fut lourdement influencée par les développements politiques, économiques et sociaux des dernières décennies.

Entre autres aspects de la vie quotidienne, le régime et les préférences alimentaires des Autochtones, de pair avec les activités d'approvisionnement en nourriture, subirent fortement l'impact des changements venus de l'extérieur-comme les politiques soviétiques de concentration des populations et les pensionnats-écoles pour les enfants. Kozlov, Nuvano et Vershubsky documentent de manière très détaillée comment de nombreux facteurs entremêlés provoquèrent des changements dans les goûts alimentaires, et comment ceux-ci ont évolué au cours de la période post-soviétique vers de nouveaux régimes alimentaires qui diffèrent de tous ceux qui les ont précédés. Pour illustrer plus spécifiquement ce phénomène, l'enquête de Yamin-Pasternak montre comment, à partir des années 1950, les peuples yupik et tchouktche, réticents au début, commencèrent à cueillir des champignons par ordre des Russes. Les Yupiget avaient plus de peurs à surmonter, puisqu'ils considéraient les champignons comme «les oreilles du diable», croyance qui allait de pair avec leur sentiment de malaise lorsqu'ils se trouvaient à l'intérieur des terres. Au fil du temps, de nombreux Autochtones en vinrent à partager la passion des Russes pour les champignons et leur cueillette. En certains cas, la rupture d'avec les anciennes croyances fut brutale, comme dans le cas de ce Yupik qui ne portait aucune attention aux avertissements de sa grand-mère, admettant candidement qu'elle ne savait pas parler le russe, tandis qu'il ne comprenait pas son yupik - rappel laconique de tout ce qui fut perdu de connaissances traditionnelles dans «une brusque scission générationnelle».

Comme l'a formulé Nikolai Vakhtin (1998: 170), «l'horrible hachoir totalitaire dans lequel les minorités de Tchoukotka se sont trouvées entre les années 1950 et 1980 pouvait amoindrir leurs aptitudes à la résistance, pouvait amenuiser les différences linguistiques et culturelles entre elles, mais il était loin d'être l'instrument qu'il fallait pour effacer ces différences (et ces peuples)». Mais quelles directions les peuples autochtones de la Tchoukotka ont-ils prises après l'effondrement du pouvoir soviétique? Dans leurs articles, Gray et Nielsen se penchent chacun de leur côté sur l'activisme autochtone et sur le rôle particulier de l'intelligentsia autochtone, et discutent des questions de l'agency, de résistance, mais aussi de passivité et de pathdependency ('dépendance au chemin parcouru') en Tchoukotka post-soviétique. Lorsqu'Aleksandr Nazarov était gouverneur de la Tchoukotka (1991-2000), l'activisme autochtone fut sapé par les autorités, comme l'établit Gray. De son côté, Nielsen, qui réalisa des enquêtes de terrain en territoire yupik à l'époque où Roman Abramovich était gouverneur (2001-2008), révèle cette «dépendance au chemin parcouru» qu'ont engendrée des décennies de paternalisme étatique, mais décrit également les initiatives naissantes des intellectuels autochtones. 
Morgounova a observé un changement éclair dans les attitudes vis-à-vis de la langue yupik dans le village de Novoye Chaplino, celles-ci étant passées de la fierté et de l'intérêt à la négligence dans l'intervalle de ses séjours, entre 2003 et 2005 . Elle considère ce changement comme une étape dans le processus de transformations postsoviétiques. Dans sa conclusion, elle soutient que le désintérêt actuel pour la langue locale doit pouvoir s'inverser, et que de tels changements sont liés au pouvoir et à l'économie. Vakhtin (2005: 145) a récemment démontré, à partir d'une longue perspective temporelle, qu'il y a déjà eu des hauts et des bas tant dans le soutien que la communauté yupik apportait à sa propre langue que dans le soutien que lui apportait l'État. Même s'il documente une situation antérieure où prévalait la négligence, à la fin des années 1940 et au début des années 1950, qui fut suivie d'un regain d'intérêt, il avance que l'indifférence des communautés pour l'avenir de leur langue, surtout lorsque celle-ci se double d'une absence de soutien de l'État, pourrait entraîner rapidement leur disparition.

La note de recherche de Rodionova sur l'enseignement de la langue yupik, dans ce volume, nous offre un point de vue local sur cette question. Il faut savoir que le yupik de Sibérie centrale parlé par les Yupiget (qui est identique à celui des habitants de l'île St-Laurent) avait de bonne heure été choisi, à l'époque soviétique, pour être l'unique langue des Esquimaux de la Tchoukotka à pouvoir être publiée et enseignée. Cette suprématie fut tardivement, et seulement partiellement, contrebalancée par la publication récente en Alaska, dans des versions anglaise et cyrillique russe, du Naukan Yupik Eskimo Dictionary (voir Jacobson 2005). Dans ce numéro également, Fortescue, de Reuse, Ranavrol'tyn et Weinstein étendent les questions linguistiques à la langue tchouktche et à ses interactions avec le yupik, ainsi qu'aux dictionnaires comparatifs intercontinentaux.

Weinstein-Tagrina et Vaté abordent chacune certains des aspects essentiels de la culture tchouktche: chansons rituelles, savoirs et représentations des rennes. Les rituels restent des facteurs d'intégration fondamentaux dans les vies des Tchouktches, bien qu'ils aient subi les assauts de ces changements évoqués dans presque toutes les contributions de ce volume. L'élevage intensif des rennes ne remonte pas au-delà de quelques siècles chez les Tchouktches, et la domestication du renne demeure une entreprise «à la limite», les animaux courant toujours le risque de retourner à l'état sauvage. Vaté décrit le système de représentations contemporain comme «un mélange subtil de changements et de continuité», expression que l'on peut appliquer à la plupart des aspects de la connaissance des modes de vie des Autochtones en Tchoukotka (voir les articles de ce volume, et Krupnik et Vakhtin 1997) et d'ailleurs en Arctique (Csonka et Schweitzer 2004).

Vaté, qui a travaillé sur le terrain en Tchoukotka pendant plusieurs années, a accepté le rôle de rédactrice invitée pour rassembler plusieurs courtes contributions d'autres chercheurs résidant en Tchoukotka. Ces derniers nous donnent un fascinant aperçu d'un milieu qui, pour les raisons qu'invoque Vaté dans son introduction, demeure insuffisamment connecté au reste du monde. Reste à espérer que les résultats de ces recherches en cours connaîtront une large diffusion. 
Les régimes politiques, les bonnes et mauvaises fortunes économiques, et, plus récemment, les gouverneurs, furent d'importants facteurs dans les changements qui ont affecté la vie des peuples de la Tchoukotka. L'objectif de ce volume est de présenter quelques-unes de ces questions, en fonction de la diversité des perspectives contemporaines. Les tendances qu'identifient certaines de ces contributions peuvent nous fournir une base pour imaginer l'avenir, mais cependant, en Tchoukotka bien plus qu'ailleurs sans doute, les lendemains restent très difficiles à entrevoir.

Parmi les nombreux collègues qui ont participé à l'élaboration de ce volume, je souhaite remercier en particulier Igor Krupnik, qui a identifié et contacté quelques-uns des auteurs potentiels, commenté plusieurs versions de cette introduction, et qui s'est avéré être un partenaire toujours prêt aux discussions stimulantes et éclairantes. Virginie Vaté doit être félicitée pour avoir rassemblé les contributions portant sur les recherches menées en Tchoukotka, et en avoir fait le suivi, malgré les difficultés de communication. Bent Nielsen a gracieusement accepté de créer toutes les cartes de cette introduction. Je remercie également Louis-Jacques Dorais et Steven Jacobson pour leurs avis concernant l'usage de certains termes. Enfin, ma gratitude va à Murielle Nagy, rédactrice de cette revue, pour son appui constant, sa patience, sa rigueur et son professionnalisme dans la préparation de ce volume.

\section{Références}

ARUTIUNOV, Sergei, Igor KRUPNIK et Mikhail CHLENOV

1982 Kitovaia alleia. Drevnosti ostrovov proliva Seniavina ('L'allée des os de baleine. Les antiquités des îles du détroit de Senyavin'), Moscow, Nauka Publishers.

ARUTIUNOV, Sergei et Dorian SERGEEV

1975 Problemy etnicheskoi istorii Beringomor'ia. Ekvenskii mogil'nik. ('Problèmes de l'histoire ethnique de la région de la mer de Béring. Le cimetière d'Ekven'). Moscow, Nauka Publishers.

BOGORAS, Waldemar [BOGORAZ, Vladimir]

1904-1909 The Chukchee, Leiden, E.J. Brill, New York, G.E. Stechert, The Jesup North Pacific Expedition, 7(1-3), Memoirs of the American Museum of Natural History, 11.

1913 The Eskimo of Siberia, E.J. Brill, New York, G.E. Stechert, The Jesup North Pacific Expedition, 8(3), Memoirs of the American Museum of Natural History, 12.

BOGOYAVLINSKY, Dmitri

1996[1989]Peoples of Russia's North: Demographic Information, in A. Pika, J. Dahl and I. Larsen (dir.), Anxious North, Copenhagen, IWGIA Document 82. 
BRONSHTEIN, Mikhail, Kirill DNEPROVSKII et Elena SUKHORUKOVA

2007 Mir arkticheskikh sveroboev ('Catalogue de l'exposition Le Monde des chasseurs maritimes de l'Arctique'), Anadyr, Moscow, Department of Culture, State Museum of Oriental Art.

CHICHLO, Boris

1981 Les Nevuqaghmiit ou la fin d'une ethnie, Études/Inuit/Studies 5(2): 29-47.

1993 Les autochtones et leur environnement: entre la colonisation et la perestroïka, in Boris Chichlo (ed.), Sibérie III: Les peuples du Kamchatka et de la Tchoukotka, Paris, Institut d'études slaves: 35-71.

CHLENOV, Mikhail

1988 Ekologicheskie faktory etnicheskoi istorii raiona Beringova proliva ('Facteurs écologiques de l'histoire ethnique de la région du détroit de Béring'), in V. Tishkov (dir.), Ekologiia amerikanskikh indeitsev $i$ eskimosov. Problemy indeanistiki, Moscow, Nauka Publishers: 64-75.

CHOINIÈRE, Robert and Benoît ROBITAILLE

1983 Évolution démographique des Inuit du Nouveau-Québec, des Territoires du Nord-Ouest, du Groenland et de l'Alaska, de 1930 à nos jours, Études/Inuit/Studies, 7(2): 125-150.

CSONKA, Yvon

1998 Tchoukotka: une illustration de la question autochtone en Russie, Recherches amérindiennes au Québec, 28(1) : 23-41.

2003 Ekven, a Prehistoric Whale Hunters' Settlement on the Asian Shore of Bering Strait, in Allen P. McCartney (dir.), Indigenous Ways to the Present: Native Whaling in the Western Arctic, Edmonton, Canadian Circumpolar Institute Press, Studies in Whaling/ Salt Lake City, University of Utah Press: 109-136.

CSONKA, Yvon, et Peter SCHWEITZER

2004 Cultures and Societies: Change and Persistence, in Arctic Human Development Report, Akureyri, Stefansson Arctic Institute: 45-68. Accessible en ligne à www.svs.is/AHDR.

DORAIS, Louis-Jacques

2004 Rectitude politique ou rectitude linguistique? Comment orthographier «Inuit» en français, Études/Inuit/Studies, 28(1): 155-159.

DUMOND, Don E.

1987 The Eskimos and Aleuts, revised edition, London, Thames and Hudson. 
DUMOND, Don E. et Richard BLAND (dir.)

2002 Archaeology in the Bering Strait Region: Research on Two Continents, Eugene, University of Oregon Anthropological Papers, 59: 227-259.

FITZHUGH, William W. et Aron CROWELL (dir.)

1988 Crossroads of Continents: Cultures of Siberia and Alaska, Washington, Smithsonian Institution.

FITZHUGH, William W. et Valérie CHAUSSONNET (dir.)

1994 Anthropology of the North Pacific Rim, Washington, Smithsonian Institution.

\section{GOSKOMSTAT ROSSII}

2004 Osnovnyi itogi vserossiyskaya perepisi naseleniya 2002 ('Résultats principaux du recensement de la population de toute la Russie en 2002'), Moscow, Goskomstat rossii.

GRØNLANDS STATISTIK

2007 Grønland 2007 statistisk årbog, Nuuk, Atuagkat.

GULLØV, Hans Christian et Robert McGHEE

2006 Did Bering Strait people initiate the Thule migration?, Alaska Journal of Anthropology, 4(1-2): 54-63.

HUGHES, Charles C.

1984 Siberian Eskimo, in David Damas (dir.), Handbook of North American Indians, volume 5, Arctic, Washington, Smithsonian Institution: 247-261.

JACOBSON, Steven A.

2005 History of the Naukan Yupik Eskimo Dictionary with implications for a future Siberian Yupik Dictionary, Études/Inuit/Studies, 29(1-2): 149-161.

KERTTULA, Anna M.

1997 Antler on the sea: Creating and maintaining cultural group boundaries among the Chukchi, Yupik, and Newcomers of Sireniki, Arctic Anthropology, 34(1): 212-226.

KRAUSS, Michael

1994 Crossroads? A twentieth-century history of contacts across the Bering Strait, in William W. Fitzhugh et Valérie Chaussonet (dir.), Anthropology of the North Pacific Rim, Washington, Smithsonian Institution: 365-379.

KRUPNIK, Igor

1987 Demograficheskoe razvitie aziatskikh eskimosov v 1970-e gody. Osnovnye tendentsii i etnosotsial'nye usloviia ('Tansitions démographiques des Esquimaux asiatiques durant les années 1970. Les principales tendances et conditions ethno-sociales'), in B. Prokhorov (dir.), Regional'nye problemy 
sotsial'no-demograficheskogo razvitiia, Moscow, Institut Sotsiologicheskikh Issledovanii: 85-110.

1993 Arctic Adaptations, Hanover and London, University Press of New England.

KRUPNIK, Igor, Yvon CSONKA et Owen MASON (dir.)

2006 The Bering Strait Universe: Cultures, Language, and History-A Tribute to Mikhail Bronshtein, special issue of the Alaska Journal of anthropology, $4(1-2)$.

KRUPNIK, Igor et William W. FITZHUGH

1995 In memoriam Richard Guy Condon, Études/Inuit/Studies, 19(2): 101-104.

KRUPNIK, Igor et William W. FITZHUGH (dir.)

2001 Gateways: Exploring the Legacy of the Jesup North Pacific Expedition, 1897-1902, Washington, Smithsonian Institution. Contributions to Circumpolar Anthropology, 1.

KRUPNIK, Igor et Nikolai VAKHTIN

1997 Indigenous knowledge in modern culture: Siberian Yupik ecological legacy in transition, Arctic Anthropology, 34(1): 236-252.

2002 In the 'House of Dismay': Knowledge, Culture, and Post-Soviet Politics in Chukotka, 1995-96, in Erich Kasten (dir.), People and the Land, Pathways to Reform in Post-Soviet Siberia, Berlin, Dietrich Reimer Verlag: 7-43.

KUNSTKAMERA

n.d. Gracheva, Galina Nikolaevna (obituary), online at htttp://web1.kunstkamera .ru/siberia/Gracheva.html.

NIELSEN, Bent

2005 Eskimoer $i$ Tjukotka: samfund og etnicitet i forandring ('Esquimaux en Tchoukotka: société et ethnicité en changement'), PhD dissertation, Copenhagen University, Copenhagen.

PIKA, Aleksandr

1987 Demograficheskaia politika $\mathrm{v}$ raionakh prozhivaniia narodov Severa: problemy i perpsectivy ('La politique démographique des aires de résidence des peuples autochtones du Nord: problèmes et perspectives'), in B. Prokhorov (dir.), Regional'nye problemy sotsial'no-demograficheskogo razvitiia, Moscow, Institut Sotsiologicheskikh Issledovanii: 43-55.

PIKA, Aleksandr et Boris PROKHOROV

1988 Bolshie problemy malykh narodov, Kommunist, 16: 76-83. (Publié en anglais, 1989, Soviet Union: The Big Problems of Small Ethnic Groups, IWGIA Newsletter, 57: 122-135, et en français, 1990, Les grands problèmes des petits peuples, Questions sibériennes, 1: 7-15.) 
PLUMET, Patrick

1996 L'esquimau: essai de synthèse de la préhistoire de l'Arctique esquimau, Revista de arqueologia americana, 10: 7-51.

POPPEL, Birger, Jack KRUSE, Gérard DUHAIME et Larisa ABRYUTINA

2007 SLiCA results, Anchorage, Institute of Social and Economic Research, accessible en ligne à www.arcticliving conditions.org.

ROBERT-LAMBLIN, Joëlle

1993 Socio-demographic situation of the Yuit and Inuit Eskimos and Chukchis of Tchoukotka, Études/Inuit/Studies, 17(2): 73-96.

SCHINDLER, Debra L.

1992 Russian hegemony and Indigenous rights in Chukotka, Études/Inuit/Studies, 16(1-2): 51-74.

1997 Redefining tradition and renegotiating ethnicity in Native Russia, Arctic Anthropology, 34(1): 194-211.

STERN, Pamela

1997 Introduction: Power, resistance, and security, Arctic Anthropology, 34(1): 16.

THOMPSON, Niobe

à paraître Settlers on the Edge: Identity and Modernization on Russia's Arctic Frontier, Vancouver, UBC Press.

VAKHTIN, Nikolai

1992 Native Peoples of the Russian Far North, London, Minory Rights Group International.

1998 Endangered languages in Northeast Siberia: Siberian Yupik and other languages of Tchoukotka, in Erich Kasten (dir.), Bicultural Education in the North, Munster, Waxmann: 159-173.

2005 Two approaches to reversing language shift and the Soviet publication program for indigenous minorities, Études/Inuit/Studies, 29(1-2): 131-147.

VAKHTIN, Nikolai et Igor KRUPNIK

1999 Power, politics, and heritage: Undercurrent transformations in the postsoviet Arctic - the case of Tchoukotka, in Frank Sejersen (dir.), Changes in the Circumpolar North, Keynotes presented at the Third International Congress of Arctic Social Sciences, Copenhagen, IASSA: 27-42. 


\title{
The Yupik people and its neighbours in Chukotka: Eight decades of rapid changes
}

\author{
Guest-edited by \\ Yvon Csonka*
}

\section{Introduction}

Études/Inuit/Studies has regularly been publishing articles on the Yupik people of Chukotka since its fifth volume in 1981, which featured contributions by Boris Chichlo and Igor Krupnik, and it has done so as recently as the 2005 volume on "Preserving language and knowledge"1. This is however the first thematic issue of the journal that features such extensive coverage of the peoples of Chukotka. The first impetus for this volume was given, of all places, in Greenland, during a meeting of the journal's editorial board in 2002, when board member and Inuit Circumpolar Council (ICC) Executive Council member Carl Christian Olsen (Puju) suggested the idea of a special issue on Chukotka. Given my previous research and continued family ties in that region, and my network of colleagues, I gladly accepted to act as its guest editor.

\section{Yupik, Chukchi, and "Chukotka"}

In Russia, Eskimosy (Eskimos) remains the officially accepted (self-)designation of peoples related to the Inuit and Yup'ik populations of the New World. Alternate designations include "Asiatic Eskimo"- the term used by Bogoras and by all of the subsequent Russian scholars in the later decades of the 20th century-, and "Siberian Eskimo" or "Siberian Yupik," the designations most popular in the United States'. Yupik (plur. Yupiget, 'real human being') is the vernacular self-designation of the Central Siberian Yupik (CSY)-speaking people of Chukotka and of St. Lawrence Island, Alaska, which is part of the USA (Figures 1 and 2). CSY-speakers and their descendants form the majority of Eskimosy within Russia. They should not be confused with the linguistically related Yup'ik (plur. Yupiit) of Western Alaska ${ }^{3}$. Chukotka is or

Department of Cultural and Social History, Ilisimatusarfik-University of Greenland, Box 1061, DK3900 Nuuk, Greenland.csonka@greennet.gl

I Two issues of this journal were partly dedicated to Chukotka and its peoples: vol. 5(2) (1981) titled "Yuit-Asiatic Eskimo," and vol. 17(2) (1993) "Chukotka, Nunavut, Nunavik."

In a reference article, Hughes (1984: 259) considers the Siberian Eskimos as a subgroup of the Asiatic Eskimos excluding the St. Lawrence Islanders. The designation Yuit (sing. Yuk, 'person') which one sometimes finds in the literature, was not used as a self-designation. adjectival form, and often as well as plural of the noun, whereas tradition has imposed the plural Inuit 
was home to other populations of Eskimo descent. The Nevuqaghmiit who were expelled from Naukan (Figure 3) in 1958 (Chichlo 1981; Krupnik and Chlenov, this volume) speak a dialect related to Central Alaskan Yup'ik, and in their language, the designation for non-Inuit Eskimos, including themselves, is Yupiit (sing. Yupik). Mikhail Chlenov, one of the contributors to this volume, once described Naukan geographically and culturally as a "third Diomede Island," insulated from the rest of the Chukchi Peninsula by the Dezhnev mountain range (Chlenov 1988: 71); and the Diomedes were in recent centuries a conduit for Alaskan influence and people into Asia, first Yup'ik, and later Inupiaq populations. In Russia the term Eskimosy also includes a small group of descendants of the Inupiat removed from Big Diomede Island in 1948, as well as former speakers of the now-extinct Sirenikski Eskimo language, which was neither Yupik nor Inupiaq. Yupiget, Yupiit and "Eskimos," therefore, are not quite the same populations. We have admitted some flexibility in the designations used by authors in this volume to accommodate precision and local custom.

Chukotka is under certain aspects a marginal area of the Inuit world today. The Eskimosy, numbering about 1,600, represent a small minority within their region, and even more so within huge Russia ${ }^{4}$. When Alaska was sold to the United States, in 1867, the Yupiget were split between two countries with very different political, cultural, and ideological systems. The separation was complete during the Cold War (1948-1988) that created barriers to local people's interaction, free travel, family and marriage ties that had thrived for generations. Furthermore, Chukotka, as a region, was isolated from the rest of the world, due to political developments in Russia, during a good part of the 20th century. The region itself (Figure 2) has been long named after more numerous neighbours of the Yupik, the Chukchi ${ }^{5}$.The present-day administrative entity was established in 1930 as the "Chukchi National Region" (okrug in Russian), and it was renamed in 1980 into the "Chukchi Autonomous Region," (Chukotskii avtonomnyi okrug) thus dropping the ethnic reference ${ }^{6}$. This remains its official name, commonly abbreviated in Russian as "Chukotka," and by extension, in English as well. The name Chukotka has become so common that it has also imposed itself in the English expression "Chukotka Autonomous Region." The region, however, looms central in early Inuit history, since it has been the cradle of the earliest Neoeskimo developments about 2000 years ago, and was critical to the origins of the Thule culture that spread to Arctic Canada and Greenland a millennium later (e.g., Csonka 2003; Dumond 1987;

as invariable adjectival, except when designating an individual (Dorais 2004: 156). This journal does not accept the term Yupik as a plural.

4 According to the Russian census of 2002, there were 1,534 "Eskimos" in Chukotka itself, and 216 more living in other parts of the Russian Federation (Goskomstat Rossii 2004). The Eskimosy represent about a tenth of the Native population of Chukotka, and they form the majority of the population in one single village, Novoye Chaplino.

5 The designation Chukchi (a plural in Russian), is supposed to derive from the self-designation of the reindeer herders among this people, whereas both maritime and inland Chukchi designate themselves as Lyg'oravetl'an ('people by excellence'). In 2002, there were 12,622 Chukchi in Chukotka itself (Goskomstat Rossii 2004).

6 Not to be confused with the geographic appendix named Chukchi Peninsula (Chukotskii Poluostrov), which is restricted roughly to the two districts of Chukotka closest to Bering Strait (Figure. 2). Most Chukotka Yupik people live on the Eastern coast of the Chukchi Peninsula (Figure 3). 


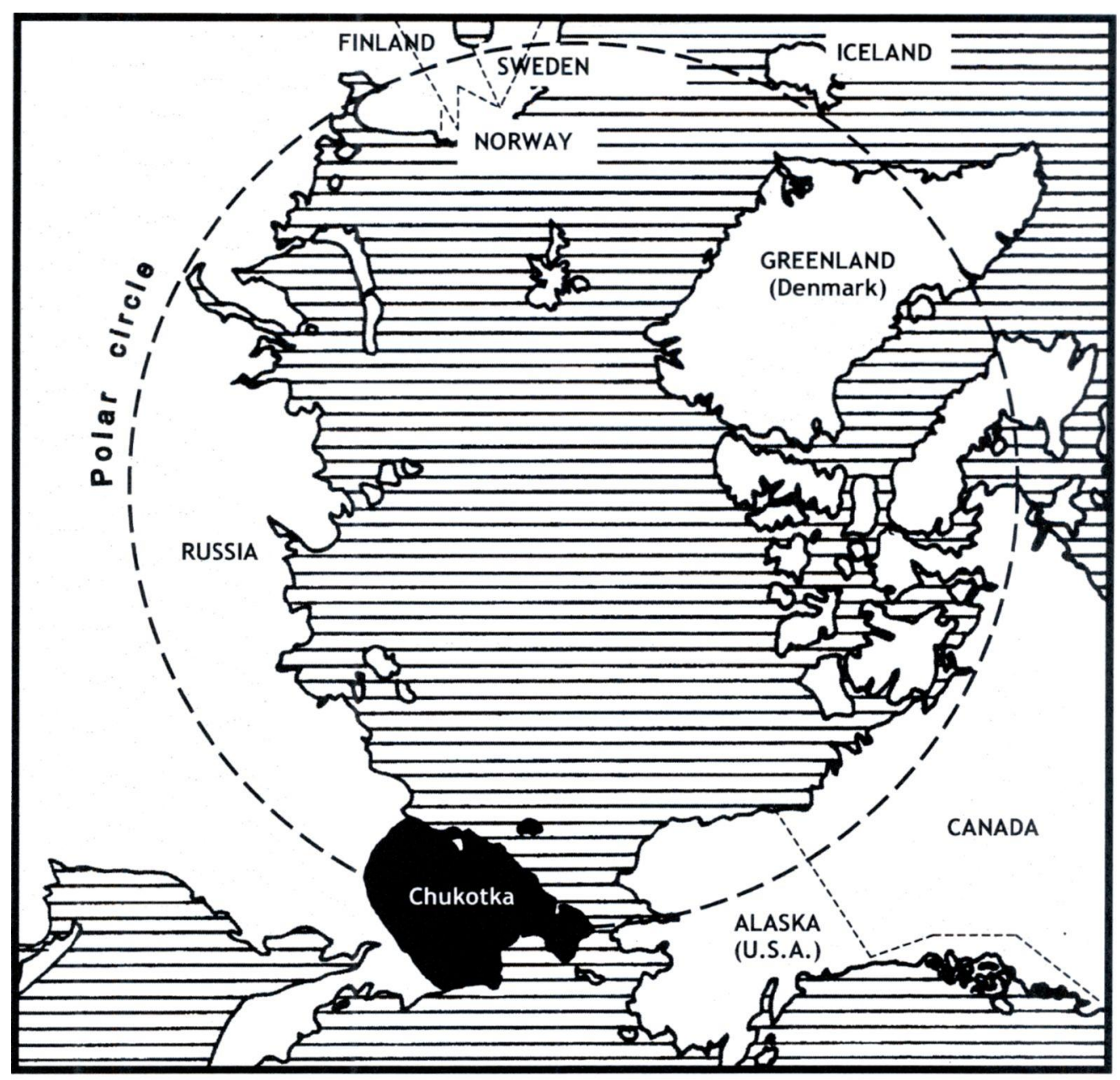

Figure 1. Map of the Arctic showing the location of Chukotka. Map by Bent Nielsen. 


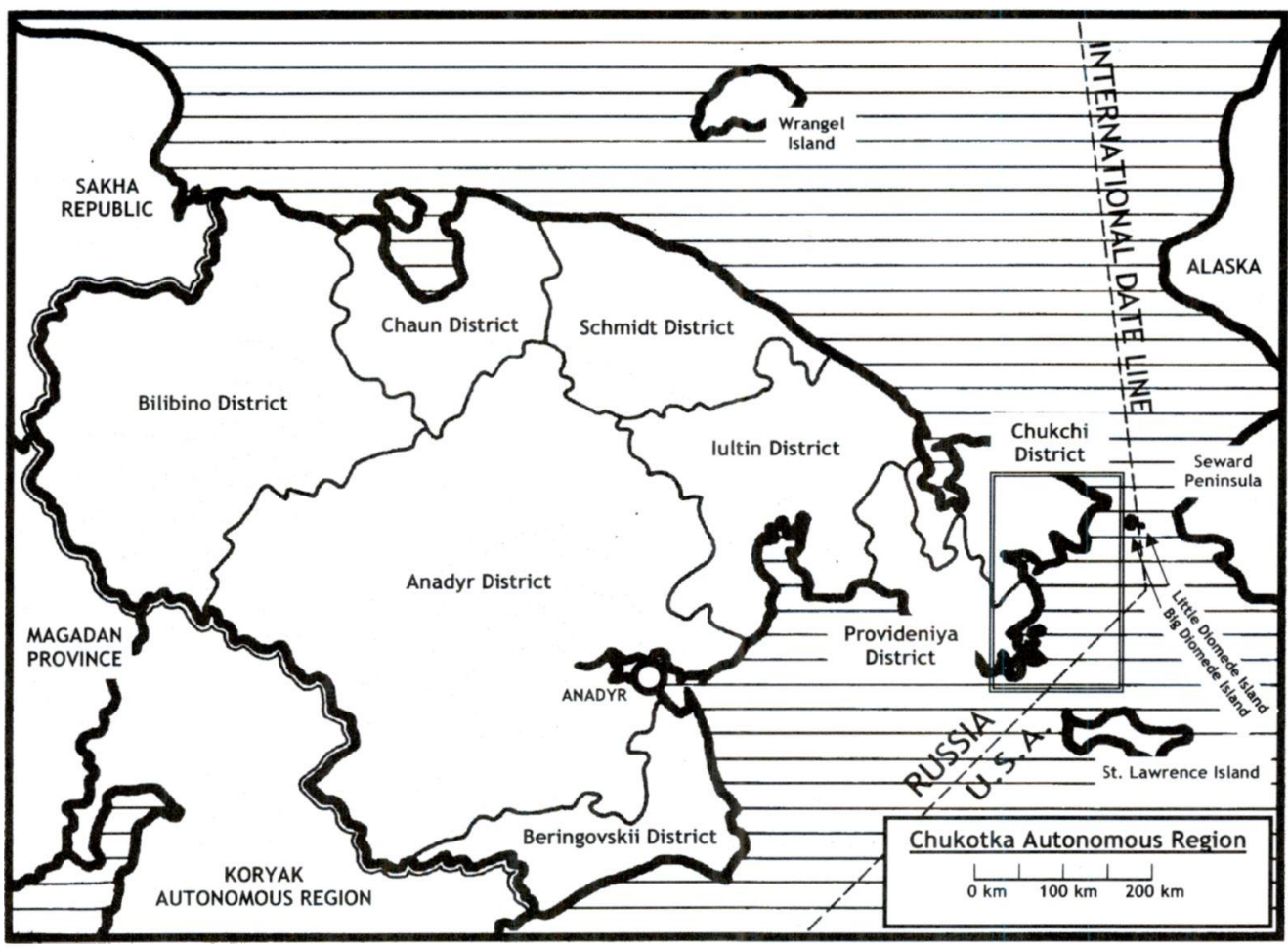

Figure 2. Map of Chukotka showing its districts and the adjacent territories. The inset shows the location of Figure 3. Map by Bent Nielsen. 


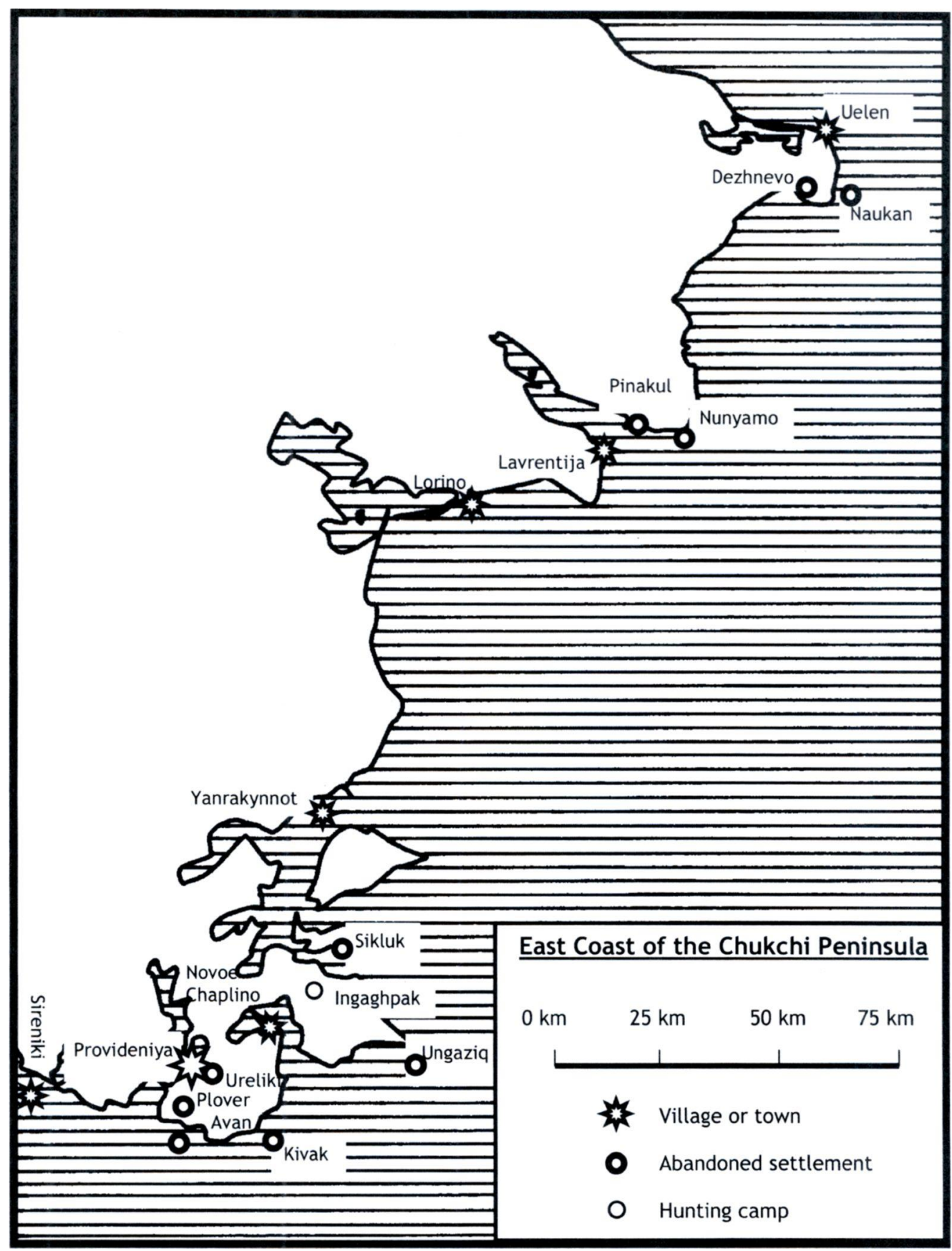

Figure 3. Map of the East coast of the Chukchi Peninsula. Map by Bent Nielsen. 
Gulløv and McGhee 2006; Plumet 1996). The latter represents the background of all subsequent historic Inuit and Yupik cultures.

In Asia even less than in any other region they have settled, the prehistoric and historic Eskimo/Yupik people were not isolated from the neighbouring populations. They were in constant contact with their various neighbours who imparted them with many cultural elements from deeper within Siberia and Asia, and also with Inupiat and Yupiit from Alaska. The Eskimo population in Asia was historically quite diverse, speaking several distinct languages and dialects. Throughout times, there were thriving exchanges between populations through trading, marriage, co-marriage, the incorporation of war prisoners as spouses or slaves, and diverse degrees of multilingualism. Russian scholars believe that the maritime Chukchi population originated from "Chukchified" Eskimo, who adopted the language and some customs of their inland neighbours, without abandoning their original economic pursuits and settlement patterns (Arutiunov et al. 1982; Arutiunov and Sergeev 1975; Bogoras 1904-1909). Also, the prehistoric and historic Eskimo had at times extended further along the Arctic and Pacific coasts of Chukotka than they do today. Such intricate cultural meetings have continued throughout the twentieth century, with the massive immigration of newcomers of many Soviet nationalities, and the Soviet policy of gathering different populations in large multi-ethnic settlements that encouraged mixed marriages, multilingualism, and rapid acculturation. Indeed, during Soviet times, Indigenous people became a minority in Chukotka: they represented $96 \%$ of the population of the region in 1926, but only less than $10 \%$ in 1989. Due to the massive outmigration of incomers, their share of the total population has since risen again to almost a third in $2002^{7}$.

\section{Post-perestroïka research in Chukotka}

This thematic issue appears on the 20th anniversary of the so-called "Friendship flight" from Nome, Alaska, to Provideniya, Chukotka. The planeload of Alaskan politicians, public officials, journalists, and St. Lawrence Island Yupik elders that landed in June 1988 in Provideniya, in the heart of the Soviet Yupik land, heralded the end of four decades of Cold War in the Bering Strait region and literally re-established contacts between Chukotka and the rest of the world (Krauss 1994). The next month, a delegation of the ICC visited the region as well. In the same period, the "Crossroads of Continents" exhibit (1988), and many subsequent scholarly publications re-emphasised the concept of a larger "North Pacific cultural area,"

The history and ethnography of the Soviet and post-Soviet immigrant population of Chukotka is chronicled in Thompson's excellent recent book (in press), which is a must-read for anyone wishing to understand political and social developments in Chukotka. Issues of ethnicity in mixed settlements during late Soviet times have been described by Kerttula (e.g., 1997) and Schindler (1992, 1997), and in post-Soviet times by Nielsen (2005) and by Vakhtin and Krupnik (1999).

The comparison of the cultures surrounding the northern rim of the Pacific was a main rationale of the Jesup North Pacific Expedition (1897-1902), directed by Franz Boas from the American Museum of Natural History in New York, that produced magistral ethnographic, folklore, and language contributions, in particular, for the Chukchi and Yupiget by Waldemar Bogoras (1904-1909, 1913). 
renewed intercontinental research collaborations (Fitzhugh and Chaussonnet 1994; Fitzhugh and Crowell 1988; Krupnik and Fitzhugh 2001).

Since the end of the Cold War, many scholars from the region, from the rest of Russia, and from other countries, have carried out human and social science field research in Chukotka at a level unseen for decades. All were confronted with considerable logistical and administrative difficulties. Tragic testimonies to these difficulties, two transportation accidents claimed the lives of colleagues. In May 1993, at the end of the "Transsibéring Expedition," a helicopter crashed on the sea ice near Enurmino, killing 8 of the 21 passengers, among them ethnographer Galina Gracheva, Russian and foreign journalists, and a representative of the expedition's sponsors (Kunstkamera n.d.) ${ }^{9}$. In September 1995, several Russian and foreign colleagues working in Chukotka-Aleksandr Pika, Steven McNabb, Richard Condon, William Richards-perished, along with local people, in the never elucidated capsizing of an open boat off Sireniki (Krupnik and Fitzhugh 1995; Stern 1997: 1). Thus, after a brief initial period of enthusiastic exchanges, Chukotka again became a remote, forbidding, and hardly less than forbidden region, which it remains to this day.

\section{Change and persistence among the peoples of Chukotka}

Given the context of longstanding extensive cultural contacts in Chukotka, it appears justified, in this thematic issue, to avoid restricting the contributions to Yupik people alone, and on the contrary, to open the discussion on what has constituted a central element of their recent history, the collective incorporation of the Indigenous peoples of Chukotka into the Soviet state, and its aftermath. In many ways, the tumultuous changes of the 20th century have set the Yupiit and Yupiget of Chukotka apart from their Inuit and Yup'ik neighbours in Alaska and elsewhere. Readers familiar with the conditions and history of Inuit peoples in other Arctic countries will however easily recognise many familiar social trends and phenomena, and realise that some of them differ in degree, though not in kind.

The comparative demographic histories of different Inuit regions provide an indicator of the poor living conditions of Native people in Chukotka: from 1926 to 2002 , the Yupik population grew by only $19 \%$, the Chukchi by $16 \%$. During comparable periods, on the other hand, the Greenlanders became more than twice more numerous, while the Canadian Inuit population grew by a factor of about six ${ }^{10}$. Yupik past demographic evolutions have been described as very dynamic, only constrained periodically by environmental factors (Krupnik 1993: chap. 7). But the near stagnation (with slight oscillations) of the population throughout the 20th century must be

9 Private funds collected in France and Switzerland in the wake of the tragedy allowed Doctors of the World (a humanitarian NGO) to run humanitarian projects aimed at relieving the plight of Chukotka Natives throughout the rest of the 1990s. These humanitarian efforts were barely tolerated by the administration of the Region.

10 Author's estimates based on census data (in Chichlo 1993; Choinière et Robitaille 1983; Grønlannds statistik 2007). These estimates do not take into account the emigration of Inuit and Yupik populations out of their region. This emigration is proportionally higher in Greenland and Canada than in Russia. 
explained by limitations other than environmental. Shifting ethnic affiliations or definitions of such, assimilation, or poor statistics are minor explaining factors. Rampant alcoholism, poor medical treatment, low mother and child health condition, and extremely high violent death rates, connected to social problems engendered by imposed changes, are the factors invoked in landmark articles by Russian specialists around the perestroïka period (Bogoyavlinsky 1996[1989]; Krupnik 1987; Pika 1987; Pika and Prokhorov 1988; Vakhtin 1992), and up to this day (Abryutina's contributions in this volume).

The papers in this volume provide a condensed panorama of the diversity of research in Chukotka over the past 10 to 20 years, and also the results of earlier contributions to the field of Yupik studies, in several disciplines ${ }^{11}$. Authors are from Chukotka itself, from the rest of Russia, from different European countries, and from North America. Many of the contributions document and analyse not so much aspects of traditional Indigenous culture and society per se, but rather the impacts of the Soviet and, later, post-Soviet regimes, as well as Indigenous adaptations and reactions to them. Such a focus is not a deliberate editorial choice, it simply constitutes evidence of the magnitude of these impacts, which have affected all Indigenous peoples of the region, and which strike many researchers as unavoidable topics of attention. The extreme rapidity of post-Soviet changes, already noted in the 1990s (e.g., Csonka 1998: 24; Vakhtin and Krupnik 1999; Krupnik and Vakhtin 2002: 7,32), continues unabated. We are therefore far from the illusion of a timeless ethnographic present, and the succession of papers in this volume reflects this time-dependent perspective.

In the "Document" section, Abryutina presents a sweeping overview of the history of the "Aboriginal peoples of Chukotka," from an insider's perspective. It was originally intended as a prologue to her presentation of the Chukotka part of the Survey of Living Conditions in the Arctic (SLiCA), which was carried out in 2005 under her leadership, and appears here among the research notes. The few comparisons of SLiCA results in different areas of the Inuit world, which she presents, show how significantly less well off Chukotka Indigenous people are, and consider themselves to be, in terms of health, consumption of country food, housing, and economic security. As an insider again, she has chosen to focus on the local context, and on free text comments by respondents, whereas more comparative tables can be found in the recently published results of this international survey (Poppel et al. 2007).

Oral historical data collected in the 1990s allow Schweitzer and Golovko to document an intriguing episode of Native religious revitalisation in Naukan in the early 1930s. In their contribution to this volume, the authors describe the context of the meetings of cultures and religions in the period surrounding this episode, and provide a theoretically insightful analysis linking it to the stress imposed by the transition from American to Russian colonialism, and especially the changes enforced by Soviet rule. Resistance in Naukan was short-lived: the village was closed by the Soviet authorities

11 This volume contains no contribution on archaeology. For recent publications on the Neoeskimo prehistory of Chukotka, see e.g., Bronshtein et al. (2007); Csonka (2003); Dumond and Bland (2002) and Krupnik et al. (2006). 
in 1958 and its access barred. Pioneers of Yupik ethnography Krupnik and Chlenov conducted fieldwork and oral history interviews with the relocatees already in the 1970s and 1980s. Here they describe how the Yupik settlement patterns in Chukotka were dislocated in the late 1950s, and this for the first time in the words, and from the points of view, of those who were displaced (previous contributions on the Naukan relocation in this journal include Chichlo[1981] and Robert-Lamblin [1993]). Although the relocations affected less than 1,000 people, and did not involve great distances, the authors show that they reverberated with particularly destructive impact on Yupik cultures and societies.

The story of the Native engraving and sculpture workshop in nearby Uelen, narrated by Bronshtein, begins at about the same time as the Naukan revitalisation movement, in 1931, and unfolds to this day. The author emphasises continuity in Native spiritual culture and artistic expression, despite the obvious fact that this venture was heavily influenced by the political, economic and social developments of the past decades.

Among other aspects of daily life, Indigenous diets and food preferences, together with food procurement activities, were heavily impacted by changes brought from the outside - such as the Soviet policies of population concentration, and of educating children in boarding schools. Kozlov, Nuvano and Vershubsky document with great detail the way many intertwined factors brought about changes in food taste, and how these evolved in the post-Soviet period towards new diets that differ from all preceding ones. As a specific illustration, Yamin-Pasternak's investigation establishes how, starting in the 1950s, Yupik and Chukchi peoples, at first reluctantly, started picking mushrooms on the instruction of Russians. Yupiget had more fears to overcome, since they considered mushrooms as the "devil's ears," a belief consistent with their unease about inland areas. Over time, many Native people came to share the Russians' passion for mushrooms and the activity of picking them. In some instances, the break with the old beliefs was abrupt, as in the case of this Yupik man who did not heed his grandmother's warnings, candidly admitting that she could not speak Russian, while he did not understand her Yupik - a sober reminder of how much traditional knowledge was lost in an "abrupt generational divide."

As Nikolai Vakhtin (1998: 170) put it, "the horrible totalitarian mincing machine in which the Chukotkan minorities found themselves in the 1950s-1980s could decrease their ability to resist, could decrease the linguistic and cultural differences between them, but was far from what is needed to wipe those differences (and those people) out." But what directions did Chukotka Indigenous people take after the Soviet power collapsed? In their articles, Gray as well as Nielsen each deal with Indigenous activism and the particular role of Indigenous intelligentsia, and discuss issues of agency, resistance, but also passivity and path-dependency in post-Soviet Chukotka. While Aleksandr Nazarov was governor of Chukotka (1991-2000), Indigenous activism was subverted by the authorities, as established by Gray. Nielsen, who conducted fieldwork in Yupik land during the term of Roman Abramovich as governor (2001-2008), reveals the "path-dependency" engendered by decades of state paternalism, but also describes fledgling initiatives by Indigenous intellectuals. 
Morgounova observed a flash shift of attitudes towards the Yupik language in the village of Novoye Chaplino, from pride and interest to neglect, between her sojourns in 2003 and 2005. She considers this shift as a stage in the process of post-soviet transformations. In her conclusion, she contends that the current disinterest in the local language may be overturned, and that such shifts are linked to power and economy. Vakhtin (2005: 145) has recently established, from a longer time perspective, that there have been ups and downs both in Yupik community support for their own language, and State support for it. Even though he documents a previous situation of local neglect, in the late 1940s-early 1950 s, followed by renewed interest, he argues that indifference of the communities for the future of their language, especially when coupled with lack of support from the State, would lead to its rapid disappearance.

Rodionova's note on Yupik language teaching, in this volume, provides a local view on the issue. Readers should be aware that the Central Siberian Yupik spoken by the Yupiget (identical to that of St. Lawrence Island residents) was chosen early on in Soviet times as the only Chukotka Eskimo language to be published and taught in. This supremacy was partly offset lately by the recent publication in Alaska, in English and Russian cyrillic versions, of a Naukan Yupik Eskimo Dictionary (see Jacobson 2005). In this issue, Fortescue, de Reuse, Ranavrol'tyn, and Weinstein, expand linguistic issues to the Chukchi language and interactions of Yupik with it, and to inter-continental comparative dictionaries.

Weinstein-Tagrina, and Vaté, each deal with some major aspects of Chukchi culture: ritual songs, and knowledge and representations of reindeer. Rituals remain a fundamental integrating factor in the lives of Chukchi, albeit they have been under siege from the changes evoked in just about every contribution to this volume. Intensive herding is not older than a few centuries among the Chukchi, and the domestication of reindeer remains a "borderline" proposition always at risk of reverting to the wild state. Vaté describes the contemporary system of representations as a "subtle mixture of changes and persistence," an expression which can apply to most aspects of Indigenous knowledge and ways of life in Chukotka (articles in this volume, Krupnik and Vakhtin 1997) and elsewhere in the Arctic (Csonka and Schweitzer 2004).

Vaté, who carried out fieldwork in Chukotka for several years, accepted to guestedit a collection of short contributions from other Chukotka-based researchers: these provide a fascinating glimpse into a milieu that, for reasons evoked in Vatés introduction, remains insufficiently connected with the rest of the world. Hopefully the results of these ongoing research projects will be made widely available.

Political regimes, economic good and bad fortunes, and in more recent times governors, have constituted important factors in the changes that have affected Chukotka peoples' lives. It is the aim of this volume to present some of the issues, from a variety of contemporary perspectives. The trends identified in some of the contributions may provide a basis for imagining the future, however, in Chukotka arguably more than in many other places, tomorrows remain particularly difficult to decipher. 
Among the many colleagues who helped with the creation of this volume, I would like to thank in particular Igor Krupnik, who identified and contacted some of the potential authors, commented several versions of this introduction, and was always a ready partner for stimulating and enlightening discussions. Virginie Vaté must be commended for having gathered and edited contributions on research carried out in Chukotka, despite difficulties in communications. Bent Nielsen has gracefully accepted to create all the maps in this introduction. I also thank Louis-Jacques Dorais and Steven Jacobson, for their advice on the use of certain terms. Finally, my gratitude goes to Murielle Nagy, editor of this journal, for her constant support, patience, thoroughness and professionalism in the preparation of this volume.

\section{References}

ARUTIUNOV, Sergei, Igor KRUPNIK and Mikhail CHLENOV

1982 Kitovaia alleia. Drevnosti ostrovov proliva Seniavina ('Whalebone Alley. Antiquities of the Senyavin Strait Islands'), Moscow, Nauka Publishers.

ARUTIUNOV, Sergei and Dorian SERGEEV

1975 Problemy etnicheskoi istorii Beringomor'ia. Ekvenskii mogil'nik. ('Problems in Ethnic History of the Bering Sea Region. The Ekven Cemetery'). Moscow, Nauka Publishers.

BOGORAS, Waldemar [BOGORAZ, Vladimir]

1904-1909 The Chukchee, Leiden, E.J. Brill, New York, G.E. Stechert, The Jesup North Pacific Expedition, 7(1-3), Memoirs of the American Museum of Natural History, 11.

1913 The Eskimo of Siberia, E.J. Brill, New York, G.E. Stechert, The Jesup North Pacific Expedition, 8(3), Memoirs of the American Museum of Natural History, 12.

BOGOYAVLINSKY, Dmitri

1996[1989]Peoples of Russia's North: Demographic Information, in A. Pika, J. Dahl and I. Larsen (eds), Anxious North, Copenhagen, IWGIA Document 82.

BRONSHTEIN, Mikhail, Kirill DNEPROVSKII and Elena SUKHORUKOVA 2007 Mir arkticheskikh sveroboev ("World of Arctic Maritime Hunters-Exhibition catalogue'), Anadyr, Moscow, Department of Culture, State Museum of Oriental Art.

CHICHLO, Boris

1981 Les Nevuqaghmiit ou la fin d'une ethnie, Études/Inuit/Studies 5(2): 29-47.

1993 Les autochtones et leur environnement: entre la colonisation et la perestroïka, in Boris Chichlo (ed.), Sibérie III: Les peuples du Kamchatka et de la Tchoukotka, Paris, Institut d'études slaves: 35-71. 
CHLENOV, Mikhail

1988 Ekologicheskie faktory etnicheskoi istorii raiona Beringova proliva ('Ecological factors of Ethnic History of the Bering Strait Region'), in V. Tishkov (ed.), Ekologiia amerikanskikh indeitsev i eskimosov. Problemy indeanistiki, Moscow, Nauka Publishers: 64-75.

CHOINIÈRE, Robert and Benoît ROBITAILLE

1983 Évolution démographique des Inuit du Nouveau-Québec, des Territoires du Nord-Ouest, du Groenland et de l'Alaksa, de 1930 à nos jours, Études/Inuit/Studies, 7(2): 125-150.

CSONKA, Yvon

1998 Tchoukotka: une illustration de la question autochtone en Russie, Recherches amérindiennes au Québec, 28(1) : 23-41.

2003 Ekven, a Prehistoric Whale Hunters' Settlement on the Asian Shore of Bering Strait, in Allen P. McCartney (ed.), Indigenous Ways to the Present: Native Whaling in the Western Arctic, Edmonton, Canadian Circumpolar Institute Press, Studies in Whaling/ Salt Lake City, University of Utah Press: 109-136.

CSONKA, Yvon, and Peter SCHWEITZER

2004 Cultures and Societies: Change and Persistence, in Arctic Human Development Report, Akureyri, Stefansson Arctic Institute: 45-68. Accessible online at www.svs.is/AHDR

DORAIS, Louis-Jacques

2004 Rectitude politique ou rectitude linguistique? Comment orthographier «Inuit» en français, Études/Inuit/Studies, 28(1): 155-159.

DUMOND, Don E.

1987 The Eskimos and Aleuts, revised edition, London, Thames and Hudson.

DUMOND, Don E. and Richard BLAND (eds)

2002 Archaeology in the Bering Strait Region: Research on Two Continents, Eugene, University of Oregon Anthropological Papers, 59: 227-259.

FITZHUGH, William W. and Aron CROWELL (eds)

1988 Crossroads of Continents: Cultures of Siberia and Alaska, Washington, Smithsonian Institution.

FITZHUGH, William W., and Valérie CHAUSSONNET (eds)

1994 Anthropology of the North Pacific Rim, Washington, Smithsonian Institution. 


\section{GOSKOMSTAT ROSSII}

2004 Osnovnyi itogi vserossiyskaya perepisi naseleniya 2002 ('Main results of the all-Russian population census of 2002'), Moscow, Goskomstat rossii.

GRØNLANDS STATISTIK

2007 Grønland 2007 statistisk årbog, Nuuk, Atuagkat.

GULLØV, Hans Christian and Robert McGHEE

2006 Did Bering Strait people initiate the Thule migration?, Alaska Journal of Anthropology, 4(1-2): 54-63.

HUGHES, Charles C.

1984 Siberian Eskimo, in David Damas (ed.), Handbook of North American Indians, volume 5, Arctic, Washington, Smithsonian Institution: 247-261.

JACOBSON, Steven A.

2005 History of the Naukan Yupik Eskimo Dictionary with implications for a future Siberian Yupik Dictionary, Études/Inuit/Studies, 29(1-2): 149-161.

KERTTULA, Anna M.

1997 Antler on the sea: Creating and maintaining cultural group boundaries among the Chukchi, Yupik, and Newcomers of Sireniki, Arctic Anthropology, 34(1): 212-226.

KRAUSS, Michael

1994 Crossroads? A twentieth-century history of contacts across the Bering Strait, in William W. Fitzhugh and Valérie Chaussonet (ed.), Anthropology of the North Pacific Rim, Washington, Smithsonian Institution: 365-379.

KRUPNIK, Igor

1987 Demograficheskoe razvitie aziatskikh eskimosov v 1970-e gody. Osnovnye tendentsii i etnosotsial'nye usloviia ('Demographic Transitions of the Asiatic Eskimos During the 1970s. Major Trends and Ethno-Social Conditions'), in B. Prokhorov (ed.), Regional'nye problemy sotsial'nodemograficheskogo razvitiia, Moscow, Institut Sotsiologicheskikh Issledovanii: 85-110.

KRUPNIK, Igor

1993 Arctic Adaptations, Hanover and London, University Press of New England.

KRUPNIK, Igor, Yvon CSONKA and Owen MASON (guest eds)

2006 The Bering Strait Universe: Cultures, Language, and History-A Tribute to Mikhail Bronshtein, special issue of the Alaska Journal of anthropology, $4(1-2)$.

KRUPNIK, Igor and William W. FITZHUGH

1995 In memoriam Richard Guy Condon, Études/Inuit/Studies, 19(2): 101-104. 
KRUPNIK, Igor and William W. FITZHUGH (eds)

2001 Gateways: Exploring the Legacy of the Jesup North Pacific Expedition, 1897-1902, Washington, Smithsonian Institution. Contributions to Circumpolar Anthropology, 1.

KRUPNIK, Igor, and Nikolai VAKHTIN

1997 Indigenous knowledge in modern culture: Siberian Yupik ecological legacy in transition, Arctic Anthropology, 34(1): 236-252.

2002 In the 'House of Dismay': Knowledge, Culture, and Post-Soviet Politics in Chukotka, 1995-96, in Erich Kasten (ed.), People and the Land, Pathways to Reform in Post-Soviet Siberia, Berlin, Dietrich Reimer Verlag: 7-43.

KUNSTKAMERA

n.d. Gracheva, Galina Nikolaevna (obituary), online at htttp://web1.kunstkamera .ru/siberia/Gracheva.html.

NIELSEN, Bent

2005 Eskimoer $i$ Tjukotka: samfund og etnicitet $i$ forandring ('Eskimos in Chukotka: Changing Society and Ethnicity'), $\mathrm{PhD}$ dissertation, Copenhagen University, Copenhagen.

PIKA, Aleksandr

1987 Demograficheskaia politika $\mathrm{v}$ raionakh prozhivaniia narodov Severa: problemy i perpsectivy ('Demographic Policy in the Areas of Residence of Northern Indigenous Peoples: problems and perspectives'), in B. Prokhorov (ed.), Regional'nye problemy sotsial'no-demograficheskogo razvitiia, Moscow, Institut Sotsiologicheskikh Issledovanii: 43-55.

PIKA, Aleksandr and Boris PROKHOROV

1988 Bolshie problemy malykh narodov, Kommunist, 16: 76-83. (Published in English, 1989, Soviet Union: The Big Problems of Small Ethnic Groups, IWGIA Newsletter, 57: 122-135, and in French, 1990, Les grands problèmes des petits peuples, Questions sibériennes, 1:7-15.)

POPPEL, Birger, Jack KRUSE, Gérard DUHAIME and Larisa ABRYUTINA

2007 SLiCA results, Anchorage, Institute of Social and Economic Research, available online at www.arcticlivingconditions.org.

ROBERT-LAMBLIN, Joëlle

1993 Socio-demographic situation of the Yuit and Inuit Eskimos and Chukchis of Chukotka, Etudes/Inuit/Studies, 17(2): 73-96.

SCHINDLER, Debra L.

1992 Russian hegemony and Indigenous rights in Chukotka, Études/Inuit/Studies, 16(1-2): 51-74. 
1997 Redefining tradition and renegotiating ethnicity in Native Russia, Arctic Anthropology, 34(1): 194-211.

STERN, Pamela

1997 Introduction: Power, resistance, and security, Arctic Anthropology, 34(1): 16 .

THOMPSON, Niobe

in press Settlers on the Edge: Identity and Modernization on Russia's Arctic Frontier, Vancouver, UBC Press.

VAKHTIN, Nikolai

1992 Native Peoples of the Russian Far North, London, Minory Rights Group International.

1998 Endangered languages in Northeast Siberia: Siberian Yupik and other languages of Chukotka, in Erich Kasten (ed.), Bicultural Education in the North, Munster, Waxmann: 159-173.

2005 Two approaches to reversing language shift and the Soviet publication program for indigenous minorities, Études/Inuit/Studies, 29(1-2): 131-147.

VAKHTIN, Nikolai and Igor KRUPNIK

1999 Power, politics, and heritage: Undercurrent transformations in the postsoviet Arctic- - the case of Chukotka, in Frank Sejersen (ed.), Changes in the Circumpolar North, Keynotes presented at the Third International Congress of Arctic Social Sciences, Copenhagen, IASSA: 27-42. 